\title{
The Natural Product 6-Gingerol Inhibits Inflammation-Associated Osteoclast Differentiation via Reduction of Prostaglandin $\mathrm{E}_{2}$ Levels
}

\author{
Youn-Hwan Hwang ${ }^{(D)}$, Taesoo Kim, Rajeong Kim and Hyunil Ha * \\ Herbal Medicine Research Division, Korea Institute of Oriental Medicine, Daejeon 34054, Korea; \\ hyhhwang@kiom.re.kr (Y.-H.H.); xotn91@kiom.re.kr (T.K.); younme1112@nate.com (R.K.) \\ * Correspondence: hyunil74@kiom.re.kr; Tel.: +82-42-868-9367
}

Received: 20 June 2018; Accepted: 14 July 2018; Published: 16 July 2018

\begin{abstract}
The natural product 6-gingerol, a major bioactive component of the rhizome of ginger (Zingiber officinale), is known to have several beneficial effects on health, including anti-inflammatory activity. The present study aimed to investigate the effects of 6-gingerol on osteoclast differentiation associated with inflammation. 6-Gingerol inhibited osteoclast differentiation in co-cultures of osteoblasts and osteoclast precursor cells in response to the pro-inflammatory cytokine, interleukin (IL)-1. However, it did not affect osteoclast precursor differentiation into osteoclasts induced by the receptor activator of nuclear factor- $\mathrm{KB}$ ligand (RANKL), a key cytokine causing osteoclast differentiation. 6-Gingerol inhibited IL-1-induced RANKL expression in osteoblasts, and the addition of RANKL to the co-cultures overcame 6-gingerol-mediated inhibition of osteoclast differentiation. It also suppressed IL-1-induced prostaglandin $\mathrm{E}_{2}\left(\mathrm{PGE}_{2}\right)$ production in osteoblasts, and the addition of exogenous $\mathrm{PGE}_{2}$ reversed 6-gingerol-mediated inhibition of IL-induced RANKL expression in osteoblasts and osteoclast differentiation in the co-cultures. We found that 6-gingerol reduced $\mathrm{PGE}_{2}$ levels by suppressing enzymatic activities of cyclooxygenase and PGE synthase, which cooperatively catalyze the conversion of arachidonic acid to $\mathrm{PGE}_{2}$. Our findings demonstrate that 6-gingerol inhibits IL-1-induced osteoclast differentiation via suppression of RANKL expression in osteoblasts though reduction of $\mathrm{PGE}_{2}$ levels, suggesting its potential use in treating inflammatory bone destruction associated with excessive $\mathrm{PGE}_{2}$ production.
\end{abstract}

Keywords: 6-gingerol; osteoclast; inflammation; interleukin-1; prostaglandin $\mathrm{E}_{2}$

\section{Introduction}

Osteoclasts, the sole bone-resorbing cells, are multinucleated giant cells derived from hematopoietic monocyte-macrophage linage cells, and have a pivotal role in the pathogenesis of inflammatory bone disorders, such as rheumatoid arthritis, periodontal diseases, and periprosthetic osteolysis [1,2]. Under inflammatory conditions, excessive expression and production of osteoclastogenic mediators, including receptor activator of NF-kB ligand (RANKL) and macrophage colony-stimulating factor (M-CSF) from osteoblasts or stromal cells and inflammatory mediators from innate and adaptive immune systems, positively enhance osteoclast precursor formation, osteoclast differentiation, and bone resorption [3]. In particular, RANKL acts as an essential modulator of osteoclast differentiation and activation by directly binding to its receptor, RANK, expressed on osteoclast precursors and mature osteoclasts [4]. Thus, down-regulation of RANKL availability via control of RANKL expression and that of its decoy receptor, osteoprotegerin (OPG), has been shown to reduce osteoclast differentiation and bone loss in inflammatory conditions [5-8].

In addition to conventional drugs and therapies, several studies have investigated phytomedicines and nutraceuticals as treatments for overcoming excessive bone loss and abolishing undesirable 
and adverse effects of conventional drugs [9,10]. In east Asian, Ayurvedic, Arabic, and African traditional medicines, the rhizome of Zingiber officinale Roscoe (ginger) has been frequently used as a natural anti-inflammatory agent and pain-reliever in musculoskeletal diseases, such as arthritis, rheumatism, and muscular aches [11,12]. In recent human and experimental animal studies, the rhizome of $Z$. officinale has been demonstrated to be effective against inflammatory bone disorders, such as rheumatoid arthritis and osteoarthritis [13-15]. The rhizome of Z. officinale has a variety of pharmacological effects corresponding to several bioactive compounds, including the gingerols. In particular, 6-gingerol $\left(\mathrm{C}_{17} \mathrm{H}_{26} \mathrm{O}_{4}\right.$; molecular weight, 294.39), a primary bioactive phenylpropanoid isolated from Z $\mathrm{Z}$. officinale, has been shown to have anti-inflammatory, anti-oxidant, anti-tumoral, anti-diabetic, and anti-obesity activities in animal models [16]. 6-Gingerol-enriched products have shown improvement in joint inflammation in an experimental arthritis model due to their anti-inflammatory property [17]. Abusarah et al. [18] have demonstrated that 6-gingerol has a therapeutic effect in osteoarthritis via protection against oxidative stress and down-regulation of pro-inflammatory mediators in vitro and in vivo. It has also been shown to stimulate osteoblast differentiation and alleviate tumor necrosis factor (TNF)- $\alpha$-induced suppression of osteoblast differentiation in osteoblast-like cells, suggesting its potential benefit in bone formation [19]. However, little is known about the effects of 6-gingerol on osteoclast differentiation under inflammatory conditions. In the present study, we aimed to investigate the effects of 6-gingerol on osteoclast differentiation; the cellular and molecular mechanisms underlying the effects of 6-gingerol on inflammation-associated osteoclast differentiation were also characterized.

\section{Results and Discussion}

\subsection{Inhibitory Effects of 6-Gingerol on Osteoclast Formation via Down-Regulation of RANKL Expression}

A variety of osteoclast differentiation assays have been developed using isolated primary cells and stable cell lines with various osteotropic factors, such as IL-1, lipopolysaccharides, parathyroid hormone, prostaglandin $\mathrm{E}_{2}\left(\mathrm{PGE}_{2}\right), \mathrm{M}-\mathrm{CSF}$, and RANKL [20]. Among the assays, we selected two systems, the co-culture of primary mouse calvarial osteoblasts (POBs) and bone marrow cells (BMCs) with IL-1, and bone marrow-derived macrophage (BMM) cultures with M-CSF and RANKL, to discriminate between the indirect and direct effects of 6-gingerol on osteoclast differentiation. IL-1 is involved in osteoclast-mediated bone destruction under inflammatory conditions, including rheumatoid arthritis $[3,21]$. In this context, we used IL-1 to induce osteoclast differentiation in the co-culture system, in which IL-1 indirectly induces osteoclast differentiation of BMCs containing osteoclast precursors via its effects on POBs. In the BMM culture system, RANKL induces osteoclast differentiation via direct action on osteoclast precursors.

Treatment with IL-1 for seven days increased tartrate-resistant acid phosphatase (TRAP) activity, a maker of osteoclast differentiation, and successfully induced osteoclast formation in co-cultures of POBs and BMCs, which was inhibited by 6-gingerol in a dose-dependent fashion (Figure 1). 6-Gingerol at concentrations higher than $10 \mu \mathrm{M}$ caused almost complete inhibition of osteoclast formation. We next investigated whether 6-gingerol directly affected the differentiation of osteoclast precursors into osteoclasts. 6-Gingerol at concentrations up to $20 \mu \mathrm{M}$ affected neither cell viability nor RANKL-induced osteoclast differentiation in BMM cultures (Figure 2). Based on the different effects of 6-gingerol in the two culture systems, we hypothesized that 6-gingerol affects the ability of POBs to support osteoclast differentiation.

IL-1 is known to enhance the osteoclast-supporting activity of POBs by up-regulating RANKL expression and down-regulating expression of its decoy receptor, OPG [5,22]. Therefore, we examined whether 6-gingerol affects IL-1-induced RANKL and OPG expression in POBs. Real-time PCR analysis and enzyme-linked immunosorbent assay (ELISA) showed that IL-1 increased RANKL mRNA and protein expression in POBs; this was inhibited by 6-gingerol in a dose-dependent manner (Figure 3A,B). In contrast, 6-gingerol did not affect IL-1-induced reduction of OPG mRNA expression 
and protein secretion. The addition of exogenous RANKL to IL-1-stimulated co-cultures of POBs and BMCs reversed the inhibitory effects of 6-gingerol on osteoclast formation (Figure 3C). These results suggested that the anti-osteoclastogenic effects of 6-ginerol did not result from cytotoxicity in the co-cultures and that 6-gingerol-mediated suppression of RANKL expression in POBs contributed to its anti-osteoclastogenic effects in the co-cultures.

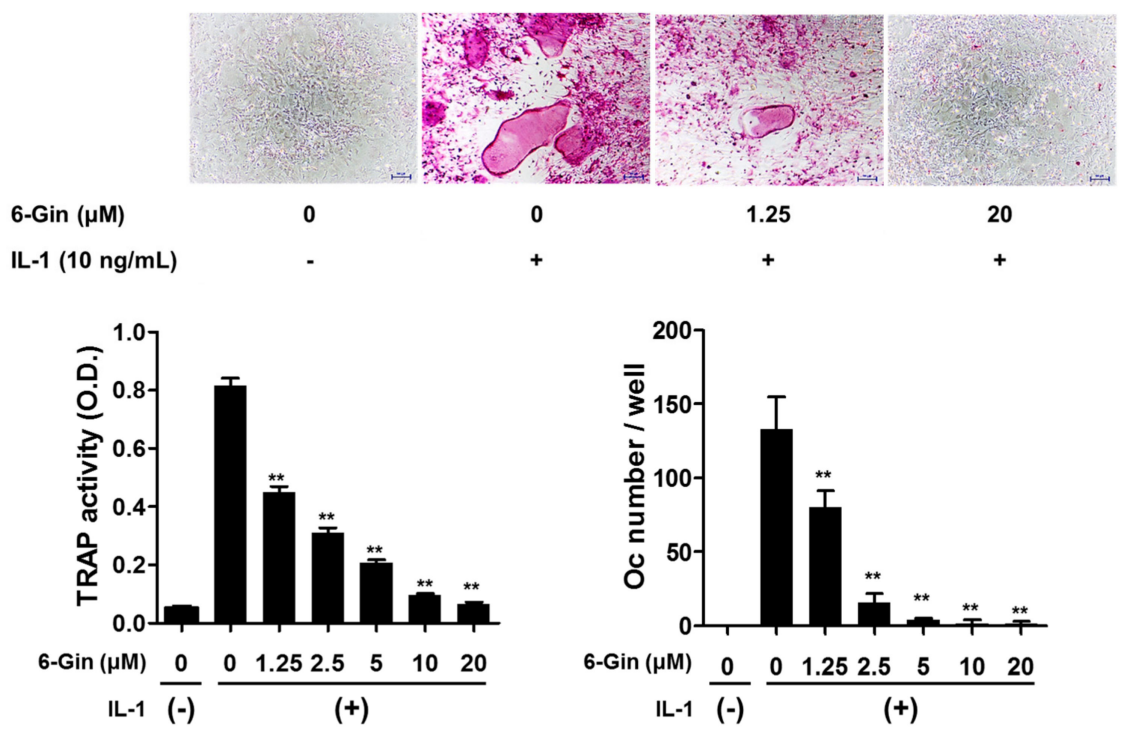

Figure 1. 6-Gingerol inhibits IL-1-induced osteoclast formation in co-cultures of primary mouse calvarial osteoblasts (POBs) and bone marrow cells (BMCs). The co-cultures were incubated with IL-1 $(10 \mathrm{ng} / \mathrm{mL})$ for seven days with 6-gingerol (6-Gin, $1.25-20 \mu \mathrm{M})$ pretreatment $1 \mathrm{~h}$ prior to IL-1 treatment. After fixation, total cellular tartrate-resistant acid phosphatase (TRAP) activity was measured, and TRAP-positive multinucleated giant cells ( $\geq$ three nuclei, $\geq 50 \mu \mathrm{m}$ in diameter) were counted as osteoclasts. ${ }^{* *} p<0.01$ vs. treatment with IL-1 alone. Scale bar $=100 \mu \mathrm{m}$.

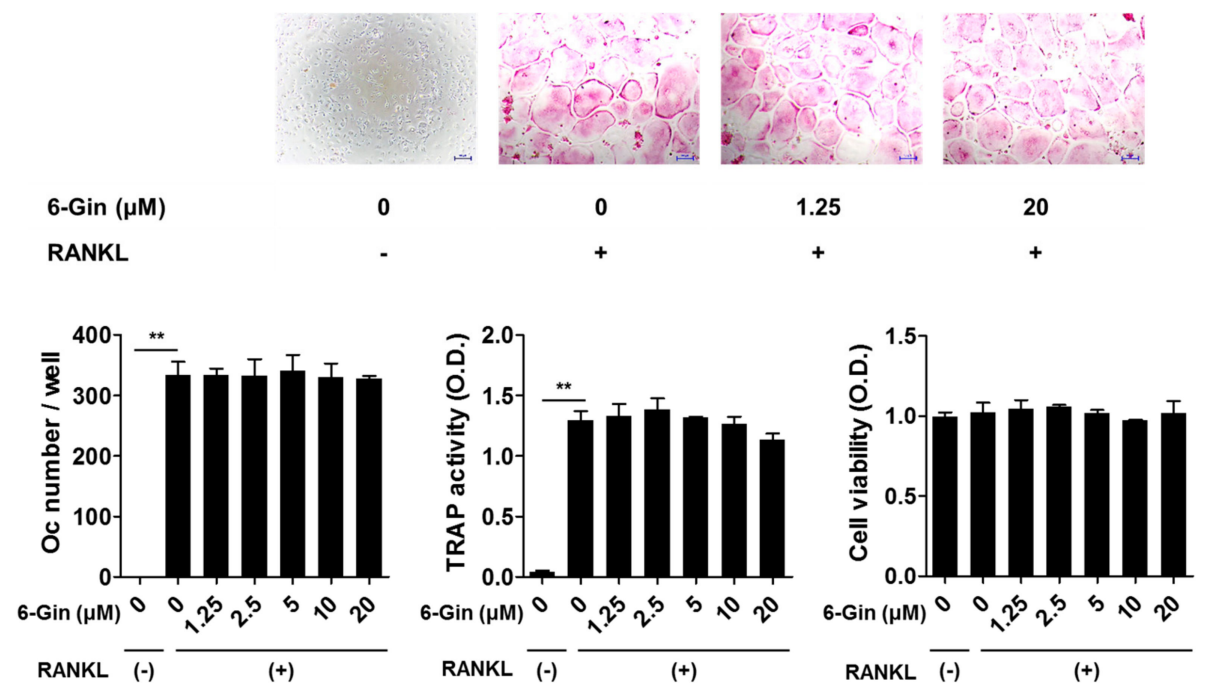

Figure 2. 6-Gingerol does not affect receptor activator of NF- $\mathrm{kB}$ ligand (RANKL)-induced osteoclast formation in bone marrow-derived macrophages (BMMs). BMMs were incubated with or without macrophage colony-stimulating factor (M-CSF) (30 ng/mL), RANKL (100 ng/mL), and 6-gingerol (6-Gin, 1.25-20 $\mu \mathrm{M}$ ) for four days. Total cellular TRAP activity and the number of osteoclasts were measured. Cell viability was determined using Cell Counting Kit-8 assay. ${ }^{* *} p<0.01$ vs. control with no RANKL treatment. Scale bar $=100 \mu \mathrm{m}$. 
(A)

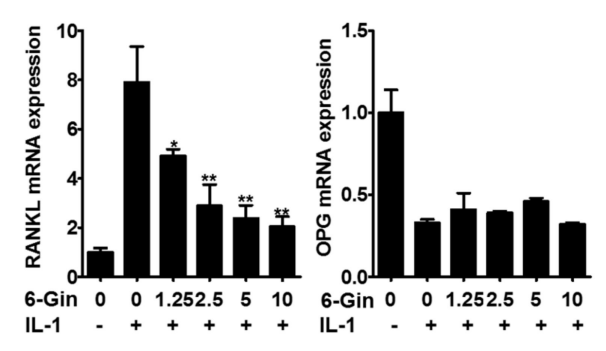

(C)

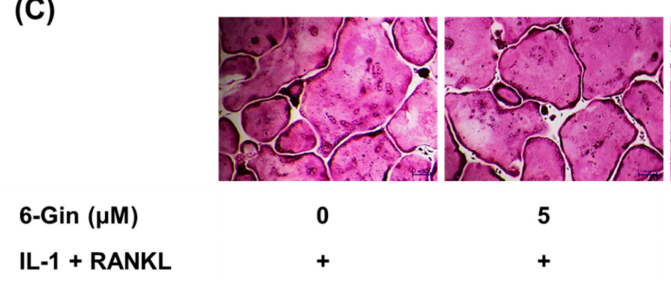

(B)
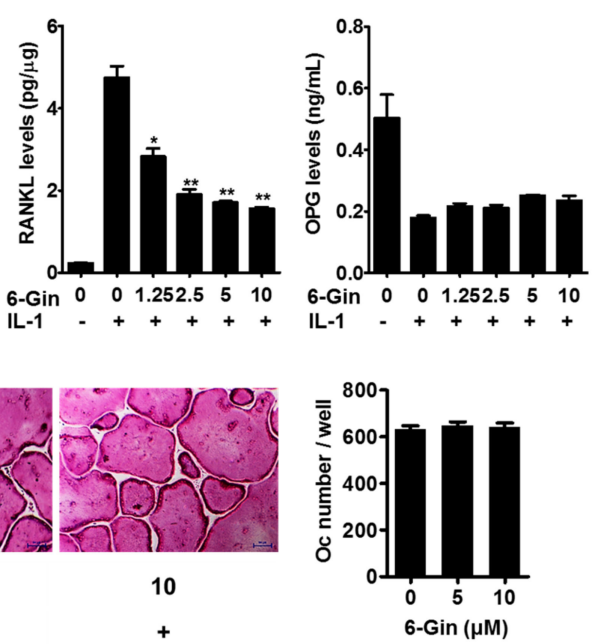

Figure 3. 6-Gingerol inhibits IL-1-induced RANKL expression in POBs. (A) POBs were incubated with or without IL-1 (10 ng/mL) and 6-gingerol (6-Gin, 1.25-20 $\mu \mathrm{M})$ for $24 \mathrm{~h}$. Gene expression levels of RANKL and osteoprotegerin (OPG) were analyzed using quantitative real-time PCR; (B) RANKL levels in POB lysates and OPG levels in the culture medium were determined using corresponding enzyme-linked immunosorbent assay (ELISA) kits; (C) POBs and BMCs were co-cultured with or without 6-gingerol (5 or $10 \mu \mathrm{M})$, and IL-1 $(10 \mathrm{ng} / \mathrm{mL})$, and RANKL $(50 \mathrm{ng} / \mathrm{mL})$ for five days. The number of osteoclasts was counted. ${ }^{*} p<0.05$ and ** $p<0.01$ vs. treatment with IL-1 alone. Scale bar $=100 \mu \mathrm{m}$.

\subsection{Inhibitory Effects of 6-Gingerol on IL-1-Induced $P G E_{2}$ Production}

We next sought to understand how 6-gingerol inhibits IL-1-induced RANKL expression in POBs. $\mathrm{PGE}_{2}$ is known to elevate RANKL expression in several cell types, including osteoblasts, and to induce osteoclast differentiation and osteoclast-mediated bone loss [7,23-25]. In addition, we previously found that $\mathrm{PGE}_{2}$ mediates IL-1-induced RANKL expression in POBs and osteoclast formation in co-cultures [5]. Therefore, we investigated whether $\mathrm{PGE}_{2}$ production was responsible for the inhibitory effects of 6-gingerol on IL-1-induced RANKL expression in POBs. IL-1 stimulated $\mathrm{PGE}_{2}$ production in POBs, which was inhibited by 6-gingerol. Inhibition of IL-1-induced RANKL protein expression by 6-gingerol was overcome by the addition of exogenous $\mathrm{PGE}_{2}$ (Figure $4 \mathrm{~A}$ ). In addition, exogenous $\mathrm{PGE}_{2}$ reversed the inhibitory effects of 6-gingerol on IL-1-induced osteoclast formation in the co-cultures (Figure 4B). These results strongly suggested that 6-gingerol inhibited IL-1-induced RANKL expression in POBs via suppression of $\mathrm{PGE}_{2}$ production, leading to inhibition of IL-1-induced osteoclast formation in the co-cultures.

We then attempted to elucidate the molecular mechanism(s) underlying 6-gingerol-mediated suppression of $\mathrm{PGE}_{2}$ production. $\mathrm{PGE}_{2}$ biosynthesis is achieved by sequential steps catalyzed by three groups of enzymes. These include the release of arachidonic acid from plasma membrane phospholipids by phospholipase A2 (PLA2) enzymes, the conversion of free arachidonic acid to $\mathrm{PGH}_{2}$ by cyclooxygenase (COX) enzymes, which is the rate-limiting step in the production of $\mathrm{PGE}_{2}$, and the final isomerization of $\mathrm{PGH}_{2}$ to $\mathrm{PGE}_{2}$ by PGE synthase (PGES) enzymes [26]. Among the isoenzymes involved in each step, cytosolic PLA2 $\alpha$ (cPLA2), COX-2, and microsomal PGES-1 (mPGES-1) have been shown to be mainly involved in $\mathrm{PGE}_{2}$ synthesis in osteoblasts and stromal cells in response to IL-1 and LPS $[5,24,27]$. It was shown that 6-gingerol does not affect cPLA2 activity even at $10 \mu \mathrm{M}$ [28]. Therefore, we examined whether 6-gingerol altered IL-1-induced expression of COX-2 and mPGES-1, which are highly induced in osteoblasts due to inflammatory stimuli $[5,27]$. Treatment of POBs with IL-1 for $24 \mathrm{~h}$ markedly increased the mRNA and protein expression of COX-2 and mPGES-1, which was not affected by 6-gingerol at concentrations below $5 \mu \mathrm{M}$ (Figure 5A). Although 6-gingerol, at a concentration of $10 \mu \mathrm{M}$, slightly suppressed IL-1-induced COX-2 mRNA expression, it did 
not affect IL-1-induced up-regulation of COX-2 protein levels. Therefore, we next investigated whether 6-gingerol affected COX/PGES activity. In POBs pretreated with IL-1 for $24 \mathrm{~h}$ to induce COX-2 and mPGES-1 expression, 6-gingerol treatment for $30 \mathrm{~min}$ inhibited IL-1-indeuced PGE 2 production. Furthermore, the addition of exogenous arachidonic acid further increased IL-1-induced $\mathrm{PGE}_{2}$ production, which was also abrogated by 6-gingerol (Figure 5B). These results suggested that 6-gingerol inhibited IL-1-induced $\mathrm{PGE}_{2}$ production by suppressing the COX/PGES-mediated conversion of arachidonic acid to $\mathrm{PGE}_{2}$. Extracellular $\mathrm{PGE}_{2}$ levels can also be regulated by other components of the COX pathway. Extracellular $\mathrm{PGE}_{2}$ is imported by the prostaglandin transporter and metabolized into an inactive form by 15-prostaglandin dehydrogenase [29]. In the present study, the addition of exogenous PGE2 completely reversed the inhibitory effects of 6-gingerol on IL-1-induced RANKL expression and osteoclast formation (Figure 4A,B), suggesting that the clearance of extracellular $\mathrm{PGE}_{2}$ was not mainly involved in 6-gingerol-induced reduction of extracellular $\mathrm{PGE}_{2}$ levels. However, further investigations are required to elucidate the detailed molecular mechanisms underlying 6-gingerol-mediated reduction of $\mathrm{PGE}_{2}$ levels.

In summary, we showed that 6-gingerol inhibits IL-1-induced osteoclast differentiation via suppression of RANKL expression in osteoblasts through reduction of $\mathrm{PGE}_{2}$ levels. In addition to such inhibitory effects on osteoclast differentiation, it has also been shown that 6-gingerol stimulates osteoblast proliferation and differentiation, and further restores osteoblast differentiation inhibited by the pro-inflammatory cytokine, TNF- $\alpha$ [19]. Our findings suggest that 6-gingerol may have beneficial effects on bone metabolism in inflammatory conditions.

(A)
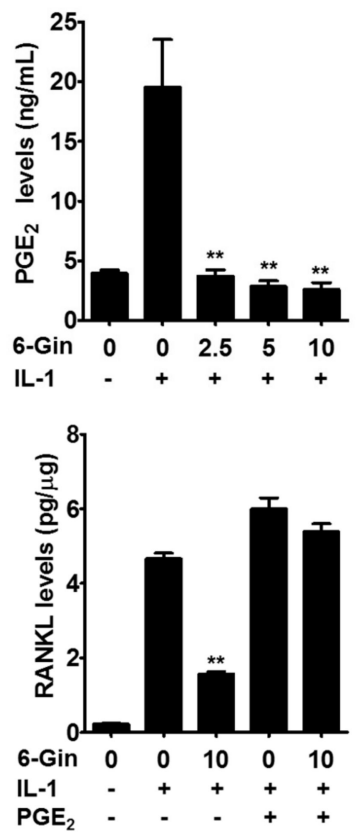

(B)
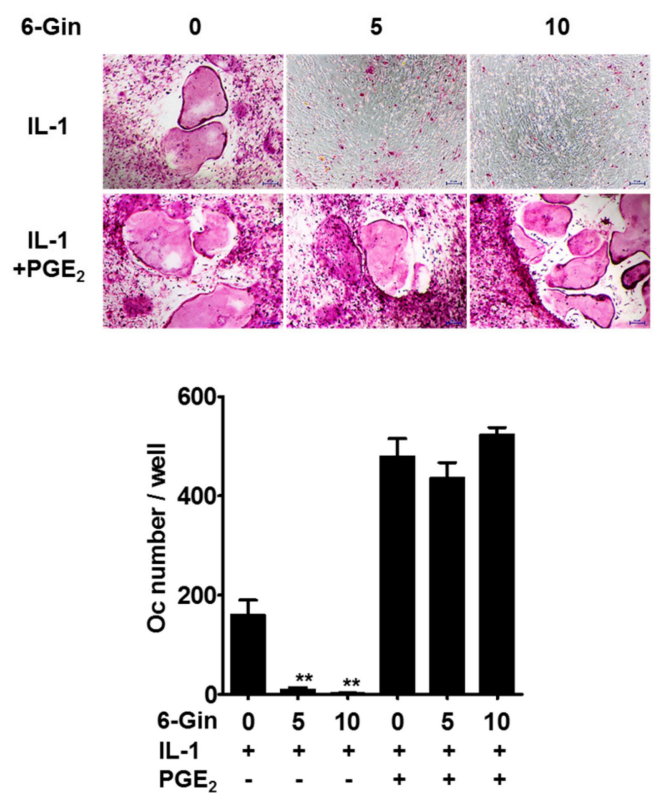

Figure 4. Prostaglandin $\mathrm{E}_{2}\left(\mathrm{PGE}_{2}\right)$ reverses the inhibitory effects of 6-gingerol on IL-1-induced osteoclast differentiation. (A) POBs were treated with or without IL-1 $(10 \mathrm{ng} / \mathrm{mL})$ and 6-gingerol (6-Gin, 2.5-10 $\mu \mathrm{M})$ for $24 \mathrm{~h}$. $\mathrm{PGE}_{2}$ levels in culture media and RANKL levels in cell lysates were determined; (B) the co-cultures of POBs and BMCs were incubated with or without 6-gingerol (5 or $10 \mu \mathrm{M}), \mathrm{IL}-1(10 \mathrm{ng} / \mathrm{mL})$, and PGE 2 $(100 \mathrm{nM})$ for seven days. The number of osteoclasts was counted. ${ }^{* *} p<0.01$ vs. treatment with IL-1 alone. Scale bar $=100 \mu \mathrm{m}$. 
(A)
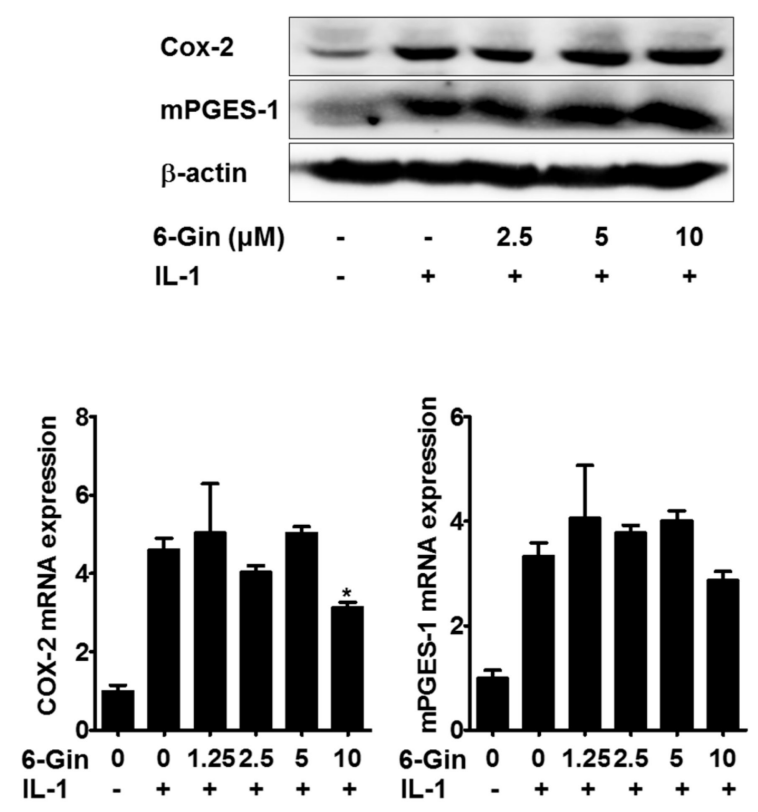

(B)

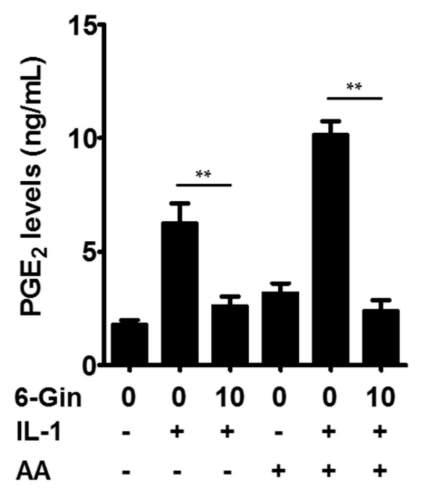

Figure 5. 6-Gingerol inhibits IL-1-induced $\mathrm{PGE}_{2}$ synthesis without affecting the expression of COX (cyclooxygenase)-2 and microsomal PGES-1 (mPGES-1). (A) POBs were treated with or without 6-gingerol (6-Gin, 1.25-10 $\mu \mathrm{M})$ and IL-1 $(10 \mathrm{ng} / \mathrm{mL})$ for $24 \mathrm{~h}$. The protein and mRNA levels of COX-2 and mPGES-1 were determined via Western blot (upper panel) and real-time PCR (lower panel); (B) POBs were treated with or without IL-1 for $24 \mathrm{~h}$, and the culture medium was replaced with fresh medium. Cells were then treated with or without 6-gingerol $(10 \mu \mathrm{M})$ for $30 \mathrm{~min}$ and further incubated with or without arachidonic acid $(\mathrm{AA}, 5 \mu \mathrm{M})$ for $15 \mathrm{~min}$. $\mathrm{PGE}_{2}$ concentrations in culture medium were determined using enzyme immunoassay (EIA). ${ }^{*} p<0.05$ vs. treatment with IL-1 alone. ${ }^{* *} p<0.01$.

\section{Materials and Methods}

\subsection{Reagents and Antibodies}

6-Gingerol and recombinant IL-1 $\alpha$ were obtained from Sigma-Aldrich (St. Louis, MO, USA) and PeproTech (Rocky Hill, NJ, USA), respectively. Recombinant soluble RANKL was prepared as reported previously [30]. M-CSF was kindly provided by Yongwon Choi (University of Pennsylvania School of Medicine). $\alpha$-Modified minimal essential medium (MEM) and fetal bovine serum (FBS) were obtained from Thermo Fisher Scientific Inc. (Rockford, IL, USA). Antibodies for COX-2 and mPGES-1 were purchased from BD Biosciences (Heidelberg, Germany) and Cayman Chemicals (Ann Arbor, MI, USA), respectively. $\beta$-actin antibody, rabbit anti-mouse IgG-horseradish peroxidase (HRP), and goat anti-rabbit IgG-HRP were obtained from Santa Cruz Biotechnology (Santa Cruz, CA, USA).

\subsection{Cell Preparation and Osteoclast Formation Assays}

The animal study was approved by the Institutional Animal Care and Use Committee of the Korea Institute of Oriental Medicine (permission numbers: 15-057 and 15-058, Daejeon, Korea). POBs were prepared from calvariae of newborn Institute of Cancer Research (ICR) mice (Samtako, Osan, South Korea) as described previously [31]. BMCs were obtained from the femurs of male ICR mice (5-7 weeks old), and BMMs were prepared from BMCs using M-CSF as described previously [31]. To determine the effects of 6-gingerol on osteoclast differentiation, we employed two different culture systems as follows; (1) co-cultures of POBs and BMCs; and (2) BMM cultures treated with M-CSF and RANKL. First, POBs $\left(2.5 \times 10^{4}\right.$ cells) and BMCs $\left(3 \times 10^{5}\right.$ cells) were co-cultured with or without IL-1 $(10 \mathrm{ng} / \mathrm{mL})$, RANKL ( $\left.50 \mathrm{ng} / \mathrm{mL}\right)$, and PGE 2 $(100 \mathrm{nM})$ in $\alpha$-MEM medium containing $10 \%$ FBS in a 48-well tissue culture plate. 6-Gingerol pre-treatment 
was done $1 \mathrm{~h}$ prior to IL-1 exposure. For the other osteoclast formation assay, BMMs $\left(1 \times 10^{4}\right.$ cells $/$ well $)$ were cultured for four days in the presence of M-CSF ( $30 \mathrm{ng} / \mathrm{mL}$ ) and RANKL $(100 \mathrm{ng} / \mathrm{mL}$ ) with or without 6-gingerol in a 96-well plate. Total cellular TRAP activity was measured colorimetrically with $p$-nitrophenyl phosphate as a substrate. TRAP staining was carried out using naphthol AS-MX phosphate and fast red violet LB salt as described previously [32]. TRAP-positive multinucleated ( $\geq$ three nuclei and $\geq 50 \mu \mathrm{m}$ of diameter) cells were considered to be osteoclasts. Cytotoxicity was measured using a Cell Counting Kit-8 per the manufacturer's instructions (Dojindo Molecular Technologies Inc., Rockville, MD, USA)

\subsection{Measurement of RANKL, OPG, and PGE2 in POBs}

POBs $\left(3 \times 10^{4}\right.$ cells/well) were cultured with or without IL-1 $(10 \mathrm{ng} / \mathrm{mL})$ for $24 \mathrm{~h}$ in a 12-well culture plate. Before IL-1 treatment, 6-gingerol treatment at concentrations of 1.25-10 $\mu \mathrm{M}$ was done. Levels of RANKL in POB lysates and those of OPG in culture media were measured using corresponding ELISA kits (R\&D systems, Minneapolis, MN, USA). PGE 2 levels in culture supernatants were determined using an enzyme immunoassay (EIA) kit (Cayman Chemicals, Ann Arbor, MI, USA).

\subsection{Measurement of COX/PGES Activity in POBs}

POBs were pretreated with or without IL-1 $(10 \mathrm{ng} / \mathrm{mL})$ for $24 \mathrm{~h}$ to induce COX-2 and mPGES-1 expression, and the culture medium was replaced with fresh medium containing different concentrations of 6-gingerol. After $30 \mathrm{~min}$ of incubation, arachidonic acid $(5 \mu \mathrm{M})$ was added for $15 \mathrm{~min}$, and $\mathrm{PGE}_{2}$ levels in the culture medium were then measured as above.

\subsection{Quantitative Real-Time PCR}

Total RNA was extracted using the RNeasy kit (Qiagen, Hilden, Germany), and cDNA was synthesized from $1 \mu \mathrm{g}$ of total RNA using the High-Capacity cDNA Reverse Transcription Kit (ABI, Waltham, MA, USA), per the manufacturer's instructions. For real-time PCR analysis, an ABI 7500 Real-Time PCR System was used in combination with TaqMan probes (Thermo Scientific, Rockford, IL, USA) and the TaqMan Universal Master Mix. In POBs, mRNA levels of RANKL (Mm0041908_m1), OPG (Mm00435452_m1), COX-2 (Mm00478374_m1), and mPGES-1 (Mm00452105_m1) were estimated, and the experiments were repeated in triplicate. The $18 \mathrm{~S}$ ribosomal gene (Hs99999901_s1) was used to normalize mRNA expression levels, and the relative expression was calculated using the $\Delta \Delta C \mathrm{t}$ method.

\subsection{Western Blot Analysis}

Protein contents in the cell lysates prepared using RIPA buffer were determined using a bicinchoninic acid assay kit (Thermo Scientific). Total protein $(40 \mu \mathrm{g})$ was resolved using $12.5 \%$ SDS-PAGE gel electrophoresis and transferred to polyvinylidene fluoride membranes. The membranes were blocked with $5 \%$ bovine serum albumin and incubated overnight at $4{ }^{\circ} \mathrm{C}$ with primary antibodies against COX-2 (1:1000), mPGES-1 (1:500), and $\beta$-actin (1:1000). The blots were then incubated with HRP-conjugated secondary antibodies and visualized using a chemiluminescence reagent (Thermo Scientific).

\subsection{Statistical Analysis}

All data were represented as mean \pm standard deviation. Experiments were repeated three times, and results from one representative experiment are shown. Data were subjected to a Student's t-test or one-way analysis of variance followed by a Dunnett's test using the software Prism (version 5.0) (San Diego, CA, USA), and a value of $p<0.05$ was considered statistically significant.

\section{Conclusions}

In the present study, we investigated the effects of 6-gingerol on osteoclast differentiation and the underlying molecular mechanisms. We show that 6-gingerol inhibits IL-1-induced osteoclast 
differentiation through down-regulation of RANKL expression in osteoblasts by suppressing PGE $_{2}$ synthesis. Given the important roles of $\mathrm{PGE}_{2}$ in inflammatory diseases, the inhibitory action of 6-gingerol on $\mathrm{PGE}_{2}$ synthesis may provide a molecular basis for its anti-inflammatory effects and its potential use in treating inflammatory bone loss.

Author Contributions: H.H. conceived and designed the experiments; R.K. and T.K. conducted experiments; Y.H.H. and H.H. contributed in data analyses and wrote the manuscript.

Acknowledgments: This work was supported by a grant (No. K18221) from the Korea Institute of Oriental Medicine, South Korea.

Conflicts of Interest: The authors declare no conflict of interest.

\section{References}

1. Wei, S.; Siegal, G.P. Mechanisms modulating inflammatory osteolysis: A review with insights into therapeutic targets. Pathol. Res. Pract. 2008, 204, 695-706. [CrossRef] [PubMed]

2. Redlich, K.; Smolen, J.S. Inflammatory bone loss: Pathogenesis and therapeutic intervention. Nat. Rev. Drug Discov. 2012, 11, 234-250. [CrossRef] [PubMed]

3. Zupan, J.; Jeras, M.; Marc, J. Osteoimmunology and the influence of pro-inflammatory cytokines on osteoclasts. Biochem. Med. 2013, 23, 43-63. [CrossRef]

4. Tanaka, S.; Nakamura, K.; Takahasi, N.; Suda, T. Role of RANKL in physiological and pathological bone resorption and therapeutics targeting the RANKL-RANK signaling system. Immunol. Rev. 2005, 208, 30-49. [CrossRef] [PubMed]

5. Ha, H.; Lee, J.H.; Kim, H.N.; Kim, H.M.; Kwak, H.B.; Lee, S.; Kim, H.H.; Lee, Z.H. Alpha-Lipoic acid inhibits inflammatory bone resorption by suppressing prostaglandin E2 synthesis. J. Immunol. 2006, 176, 111-117. [CrossRef] [PubMed]

6. Wang, J.; Wu, X.; Duan, Y. Magnesium Lithospermate B Protects against Lipopolysaccharide-Induced Bone Loss by Inhibiting RANKL/RANK Pathway. Front. Pharmacol. 2018, 9, e64. [CrossRef] [PubMed]

7. Suda, K.; Udagawa, N.; Sato, N.; Takami, M.; Itoh, K.; Woo, J.T.; Takahashi, N.; Nagai, K. Suppression of osteoprotegerin expression by prostaglandin E2 is crucially involved in lipopolysaccharide-induced osteoclast formation. J. Immunol. 2004, 172, 2504-2510. [CrossRef] [PubMed]

8. Kulkarni, R.N.; Bakker, A.D.; Everts, V.; Klein-Nulend, J. Mechanical loading prevents the stimulating effect of IL-1beta on osteocyte-modulated osteoclastogenesis. Biochem. Biophys. Res. Commun. 2012, 420, 11-16. [CrossRef] [PubMed]

9. Che, C.T.; Wong, M.S.; Lam, C.W. Natural Products from Chinese Medicines with Potential Benefits to Bone Health. Molecules 2016, 21, 239. [CrossRef] [PubMed]

10. Putnam, S.E.; Scutt, A.M.; Bicknell, K.; Priestley, C.M.; Williamson, E.M. Natural products as alternative treatments for metabolic bone disorders and for maintenance of bone health. Phytother. Res. 2007, 21, 99-112. [CrossRef] [PubMed]

11. Ali, B.H.; Blunden, G.; Tanira, M.O.; Nemmar, A. Some phytochemical, pharmacological and toxicological properties of ginger (Zingiber officinale Roscoe): A review of recent research. Food Chem. Toxicol. 2008, 46, 409-420. [CrossRef] [PubMed]

12. Baliga, M.S.; Haniadka, R.; Pereira, M.M.; D'Souza, J.J; Pallaty, P.L.; Bhat, H.P.; Popuri, S. Update on the chemopreventive effects of ginger and its phytochemicals. Crit. Rev. Food Sci. Nutr. 2011, 51, 499-523. [CrossRef] [PubMed]

13. Funk, J.L.; Frye, J.B.; Oyarzo, J.N.; Chen, J.; Zhang, H.; Timmermann, B.N. Anti-Inflammatory Effects of the Essential Oils of Ginger (Zingiber officinale Roscoe) in Experimental Rheumatoid Arthritis. Pharma. Nutr. 2016, 4, 123-131. [CrossRef] [PubMed]

14. Niempoog, S.; Pawa, K.K.; Amatyakul, C. The efficacy of powdered ginger in osteoarthritis of the knee. J. Med. Assoc. Thai 2012, 95, 59-64.

15. Paramdeep, G. Efficacy and tolerability of ginger (Zingiber officinale) in patients of osteoarthritis of knee. Indian J. Physiol. Pharmacol. 2013, 57, 177-183. [PubMed]

16. Semwal, R.B.; Semwal, D.K.; Combrinck, S.; Viljoen, A.M. Gingerols and shogaols: Important nutraceutical principles from ginger. Phytochemistry 2015, 117, 554-568. [CrossRef] [PubMed] 
17. Funk, J.L.; Frye, J.B.; Oyarzo, J.N.; Timmermann, B.N. Comparative effects of two gingerol-containing Zingiber officinale extracts on experimental rheumatoid arthritis. J. Nat. Prod. 2009, 72, 403-407. [CrossRef] [PubMed]

18. Abusarah, J.; Benabdoune, H.; Shi, Q.; Lussier, B.; Martel-Pelletier, J.; Malo, M.; Fernandes, J.C.; de Souza, F.P.; Fahmi, H.; Benderdour, M. Elucidating the Role of Protandim and 6-Gingerol in Protection Against Osteoarthritis. J. Cell Biochem. 2017, 118, 1003-1013. [CrossRef] [PubMed]

19. Fan, J.Z.; Yang, X.; Bi, Z.G. The effects of 6-gingerol on proliferation, differentiation, and maturation of osteoblast-like MG-63 cells. Braz. J. Med. Biol. Res. 2015, 48, 637-643. [CrossRef] [PubMed]

20. Marino, S.; Logan, J.G.; Mellis, D.; Capulli, M. Generation and culture of osteoclasts. Bonekey Rep. 2014, 3, e570. [CrossRef] [PubMed]

21. Ruscitti, P.; Cipriani, P.; Carubbi, F.; Liakouli, V.; Zazzeroni, F.; Di Benedetto, P.; Berardicurti, O.; Alesse, E.; Giacomelli, R. The role of IL-1beta in the bone loss during rheumatic diseases. Med. Inflamm. 2015, 2015, e782382. [CrossRef] [PubMed]

22. Baek, J.M.; Cheon, Y.H.; Kwak, S.C.; Jun, H.Y.; Yoon, K.H.; Lee, M.S.; Kim, J.Y. Claudin 11 regulates bone homeostasis via bidirectional EphB4-EphrinB2 signaling. Exp. Mol. Med. 2018, 50, e50. [CrossRef] [PubMed]

23. Suzawa, T.; Miyaura, C.; Inada, M.; Maruyama, T.; Sugimoto, Y.; Ushikubi, F.; Ichikawa, A.; Narumiya, S.; Suda, T. The role of prostaglandin E receptor subtypes (EP1, EP2, EP3, and EP4) in bone resorption: An analysis using specific agonists for the respective EPs. Endocrinology 2000, 141, 1554-1559. [CrossRef] [PubMed]

24. Miyaura, C.; Inada, M.; Matsumoto, C.; Ohshiba, T.; Uozumi, N.; Shimizu, T.; Ito, A. An essential role of cytosolic phospholipase A2alpha in prostaglandin E2-mediated bone resorption associated with inflammation. J. Exp. Med. 2003, 197, 1303-1310. [CrossRef] [PubMed]

25. Tsutsumi, R.; Xie, C.; Wei, X.; Zhang, M.; Zhang, X.; Flick, L.M.; Schwarz, E.M.; O'Keefe, R.J. PGE2 signaling through the EP4 receptor on fibroblasts upregulates RANKL and stimulates osteolysis. J. Bone Miner. Res. 2009, 24, 1753-1762. [CrossRef] [PubMed]

26. Harris, S.G.; Padilla, J.; Koumas, L.; Ray, D.; Phipps, R.P. Prostaglandins as modulators of immunity. Trends Iimmunol. 2002, 23, 144-1450. [CrossRef]

27. Inada, M.; Matsumoto, C.; Uematsu, S.; Akira, S.; Miyaura, C. Membrane-bound prostaglandin E synthase1-mediated prostaglandin E2 production by osteoblast plays a critical role in lipopolysaccharide-induced bone loss associated with inflammation. J. Immunol. 2006, 177, 1879-1885. [CrossRef] [PubMed]

28. Nievergelt, A.; Marazzi, J.; Schoop, R.; Altmann, K.H.; Gertsch, J. Ginger phenylpropanoids inhibit IL-1beta and prostanoid secretion and disrupt arachidonate-phospholipid remodeling by targeting phospholipases A2. J. Immunol. 2011, 187, 4140-4150. [CrossRef] [PubMed]

29. Nomura, T.; Lu, R.; Pucci, M.L.; Schuster, V.L. The two-step model of prostaglandin signal termination: In vitro reconstitution with the prostaglandin transporter and prostaglandin 15 dehydrogenase. Mol. Pharmacol. 2004, 65, 973-978. [CrossRef] [PubMed]

30. Tomimori, Y.; Mori, K.; Koide, M.; Nakamichi, Y.; Ninomiya, T.; Udagawa, N.; Yasuda, H. Evaluation of pharmaceuticals with a novel 50-h animal model of bone loss. J. Bone Miner. Res. 2009, 24, 1194-1205. [CrossRef] [PubMed]

31. Ha, H.; Shim, K.S.; Kim, T.; Lee, C.J.; Park, J.H.; Kim, H.S.; Ma, J.Y. Water extract of the fruits of Alpinia oxyphylla inhibits osteoclast differentiation and bone loss. BMC Complement. Altern. Med. 2014, 14, e352. [CrossRef] [PubMed]

32. Ha, H.; An, H.; Shim, K.S.; Kim, T.; Lee, K.J.; Hwang, Y.H.; Ma, J.Y. Ethanol extract of Atractylodes macrocephala protects bone loss by inhibiting osteoclast differentiation. Molecules 2013, 18, 7376-7388. [CrossRef] [PubMed]

(C) 2018 by the authors. Licensee MDPI, Basel, Switzerland. This article is an open access article distributed under the terms and conditions of the Creative Commons Attribution (CC BY) license (http:/ / creativecommons.org/licenses/by/4.0/). 